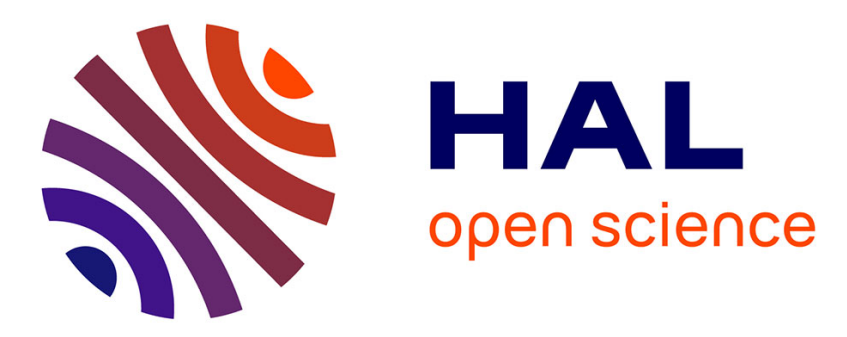

\title{
La ville dans la montagne, la montagne comme une ville: analogies architecturales et urbaines dans la représentation des Alpes chez les écrivains voyageurs aux XVIIIe et XIXe siècles
}

\author{
Alain Guyot
}

\section{To cite this version:}

Alain Guyot. La ville dans la montagne, la montagne comme une ville: analogies architecturales et urbaines dans la représentation des Alpes chez les écrivains voyageurs aux XVIIIe et XIXe siècles. Revue de Géographie Alpine / Journal of Alpine Research, 1999, 87 (1), pp.51-60. 10.3406/rga.1999.2918 . hal-02358040

\author{
HAL Id: hal-02358040 \\ https://hal.science/hal-02358040
}

Submitted on 11 Nov 2019

HAL is a multi-disciplinary open access archive for the deposit and dissemination of scientific research documents, whether they are published or not. The documents may come from teaching and research institutions in France or abroad, or from public or private research centers.
L'archive ouverte pluridisciplinaire HAL, est destinée au dépôt et à la diffusion de documents scientifiques de niveau recherche, publiés ou non, émanant des établissements d'enseignement et de recherche français ou étrangers, des laboratoires publics ou privés. 
La ville dans la montagne, la montagne comme une ville : analogies architecturales et urbaines dans la représentation des Alpes chez les écrivains voyageurs aux XVIIle et XIXe siècles

M. Alain Guyot

\section{Citer ce document / Cite this document :}

Guyot Alain. La ville dans la montagne, la montagne comme une ville : analogies architecturales et urbaines dans la représentation des Alpes chez les écrivains voyageurs aux XVIIle et XIXe siècles. In: Revue de géographie alpine, tome 87, n¹, 1999. pp. 51-60;

doi : https://doi.org/10.3406/rga.1999.2918

https://www.persee.fr/doc/rga_0035-1121_1999_num_87_1_2918

Fichier pdf généré le 22/04/2018 


\begin{abstract}
Abstract : The eighteenth and nineteenth century descriptions of high mountain landscapes are rich in metaphors and analogies with the world of architecture. What is the reason for such numerous and regular analogies ? What role do they play ? Even though their ornamental value cannot be excluded, it remains secondary. Are such analogies intended to familiarise readers with an unknown yet not too disconcerting reality, to lessen the shock of something which is new ? Did travellers during the periods of Enlightenment and romanticism, most of whom were urban dwellers, project onto this sublime and yet inhospitable environment the imaginary elements of an urban landscape which at that time was developing rapidly and becoming accepted in French literature?
\end{abstract}

\title{
Résumé
}

La ville dans la montagne, la montagne comme une ville : analogies architecturales et urbaines dans la représentation des Alpes chez les écrivains voyageurs aux XVIIle et XIXe siècles The town in mountain areas, and mountains as towns : architectural and urban analogies in the representation of the Alps by travel writers in the eighteenth and nineteenth centuries Alain Guyot Résumé : A la lecture des descriptions des paysages de la haute montagne aux XVIIle et XIXe siècles, on ne peut qu'être frappé par la prolifération des métaphores et des comparaisons renvoyant à l'univers de l'architecture. Quel est le motif de la présence de ces analogies si nombreuses et si constantes ? Quelles peuvent en être les fonctions? Si leur valeur ornementale ne peut être exclue, elle reste secondaire : sont-elles destinées à familiariser le lecteur avec un réel inconnu de lui et par trop déconcertant, à amortir le choc de la nouveauté ? Les voyageurs des Lumières et du romantisme, citadins pour la plupart, projettent-ils sur cet environnement à la fois sublime et inhospitalier les marques imaginaires d'un paysage urbain alors en pleine expansion, au moment même où les villes acquièrent plein droit de cité en littérature ? 


\title{
La ville dans la montagne, la montagne comme une ville : analogies architecturales et urbaines dans la représentation des Alpes chez les écrivains voyageurs auX $X V I^{\mid e}$ et $X I X$ siècles
}

\author{
Alain Guyot \\ Université Stendhal Grenoble III UFR de Lettres - 1180 avenue Centrale - BP 25 F38040 Grenoble cedex 9
}

L e regain d'intérêt pour la montagne qui se dessine dans les années 1730 a déjà fait l'objet d'études nombreuses et détaillées ', et l'on n'y reviendra pas ici. Il suffit de rappeler la floraison du genre du récit de voyage dans les Alpes à partir des années 1750 et, en particulier, de "l'invention" de la vallée de Chamonix et du mont Blanc par les Anglais Windham et Pococke en $1741^{2}$. Que le voyageur traverse la Suisse pour se rendre en Italie, qu'il passe les cols du mont Cenis, du Cirand-Saint-Bernard, du Brenner, ou bien qu'il effectue le voyage de Chamonix pour aller admirer ce que l'on appelait alors les "glacières" de Savoic., il ne manque pas en tous les cas de retranscrire les "horribles splendeurs" que la nature alpestre lui révèle à foison et qu'une sensibilité nouvelle, marquée au sceau du sublime depuis les textes fondateurs de Burnet et d'Addison, de Shaftesbury et de Burke ${ }^{4}$, l'autorise enfin à reconnaître.

Si ce voyageur du XVIII" siècle finissant n'a souvent de regard que pour le spectacle naturel qui l'environne, il semble toutefois éprouver quelque peine à en rendre compre: combien de fausses prétéritions qui sont autant de véritables aveux d'impuissance face à des réalités qui dépassent bien souvent l'entendement humain ! Il n'est que de rappeler les difficultés qu'éprouve Windham à appréhender les paysages du mont Blanc, au point qu'il se réfugie bien souvent dans le récit de son expédition pour éviter de représenter la nature qui l'entoure: il faudrait en effet à ses yeux l'union d'une "imagination

\footnotetext{
1. On rappellera en particulier celles de John Grand-Carteret (La Montagne à travers les âges [1903-04], Genève-Paris, Slatkine repr., 1983, 2 vol.). Claire-Eliane ENGEL (La Littérature alpestre en France et en Angleterre aux XVIII ${ }^{*}$ et $X I X$ " siècles, Chambéry, Dardel, 1930). Claudine LACOSTE-VeYsSEYre (Les Alpes romantiques. Le thème des Alpes dans la littérature française de 1800 à 1850. Genève. Slatkine, 1981, 2 vol.). Jean LACRoxx (L'évolution du sentiment de la montagne dans la littérature, des Lumières au Romantisme, Le Monde Alpin et Rhodanien 1-2, Grenoble, 1988) et Alain Roger (Court Traité du paysage, Paris, Gallimard-NRF, 1997, p. 83 et suiv.).

2. Cf. à ce sujet Philippe Joutard, L'Invention du mont Blanc, Paris, Gallimard-Julliard, 1986.

3. " On appelle Glacieres, les montagnes qui sont le point de réunion des glaces : on a donné le nom de Glaciers aux rameaux de glace qui en dérivent. " (Ramond de Carbonnieres trad., Lettres de $M$. William Coxe à M. W. Melmoth sur l'état politique, civil et naturel de la Suisse, traduites de l'anglois, et augmentées des observations faites dans le même pays, par le traducteur, Paris, Belin, 1781, t. II, "Observations du traducteur sur les glacières et les glaciers ", note 1 de la p. 96). 4. Cf. Baldine SAInt Girons, Fiat lux. Une philosophie du sublime, Paris, Quai Voltaire-Edima-Vrin diff., 1993, p. 57 et suiv.
} 
poétique " et d'un solide "goût de la peinture " pour décrire les beautés des Alpes. Rien d'étonnant alors à ce qu'il déclare à son correspondant :

Je vous avouc que je suis extrêmement embarrassé à vous en donncr une idée juste, ne connaissant, de tout ce que j'ai vu, rien qui y ait le moindre rapport ${ }^{5}$.

Saussure ne dira pas autre chose, lorsqu'il écrira, du milieu des glaciers:

[...] comment peindre à l'imagination des objets qui n'ont rien de commun avec tout ce que l'on voit dans le reste du monde [?] Fe quand on se rappelle la belle végétation et les charmants paysages que l'on a vus les jours précédents dans les basses vallées, on est tenté de croire qu'on a été subitement transporté dans un autre monde oublié par la naturét.

On peut donc parler sans crainte d'une véritable impréparation des voyagcurs de l'époque, qu'ils soient géographes, géologues, naturalistes ou simples amateurs, à décrire les paysages de haute montagne qu'ils rencontrent, en particulier ces fameuses glacières qui constituent bien souvent la motivation même de leur voyage. Ils se trouvent en effet confrontés à une véritable "carence lexicale ", qui a été récemment analysée par les historiens des sciences ${ }^{7}$, mais qu'avait déjà fort bien perçu en son temps Bernardin de SaintPierre, dans son Voyage à l'île de France (1773):

L'art de rendre la nature est si nouveau que les termes mêmes n'en sont pas inventés. Essayez de faire la description d'une montagne de manière à la faire reconnaitre : quand vous aure\% parlé de la base, des flancs et du sommet, vous aurez tout dit! Mais que de variétés dans ces formes bombées, arrondies, allongées, aplaties, cavées, etc. ! Vous ne trouve\% que des périphrases. C'est la même difficulté pour les plaines et les vallons. Qu'on ait à décrire un palais, ce n'est plus le même embarras. On le rapporte à un ou plusicurs des cinq ordres : on le subdivise en soubassement, en corps principal, en entablement; ct dans chacune de ces masses, depuis le socle jusqu'à la corniche, il n'y a pas une moulure qui n'ait son nom ${ }^{8}$.

C'est peut-être à cette prépondérance de l'ordre architectural dans la perception esthétique que l’on doit les nombreuses analogies empruntées au lexique de l'art de Vitruve par les voyageurs alpins des Lumières finissantes, lorsqu'ils s'essayent à approcher, à travers l'écriture, des paysages réputés indescriptibles. Mais si l'on ne peut qu'abonder dans le sens de Jean Lacroix lorsqu'il souligne la pauvreté du vocabulaire de l'époque, incapable de "rendre [...] toute la complexité et toute l'exubérance d'un monde saturé de volumes, de formes, de lignes, d'arêtes ", on le suivra moins volontiers lorsqu'il qualifie la périphrase de " palliatif dérisoire" qui "signe[rait] un aveu d'impuissance" ". S'il est vrai que certains naturalistes, comme Grüner ${ }^{10}$, font preuve d'une incontestable plati-

5. Cité dans Cl.-E. Engel, Le mont Blanc vu par les écrivains et les alpinistes, Paris, Editions d'art et d'histoire-Plon, 1965 , p. 28 ; cf. aussi Ph. Joutaro, ouvr. cité, p. 91.

6. Premières Ascensions au mont Blanc 1774-1787(anthologie précédée d'une introduction de Roger CANAC), Paris, La Découverte, 1991, pp. 87-88.

7. Cf. en particulier Michèle DucHet, Anthropologie et Histoire au siècle des lumières, Paris, Maspero, 1971, p. 112, et Numa Broc, La Géographie des philosophes. Géographes et voyageurs français au XVII' siècle, thèse, Université de Lille III, 1972, p. 687.

8. Yves BÉnOt éd., Paris, La Découverte, 1983, p. 254.

9. Art. cité, p. 212.

10. Histoire naturelle des glacières de Suisse (1760 ; trad. fr. par de KéRALIo), Paris, Pancoucke, 1770. 
tude dans leurs descriptions des monts et des glaciers, on ne peut en dire autant de ceux que l'on considère comme les premiers explorateurs des Alpes - pensons en parriculier aux voyageurs dans la vallée de Chamonix. Beaucoup manifestent en effet une remarquable inventivité linguistique: en dehors de quelques emprunts au patois local (comme le mot "moraine") ou de quelques images tellement aveuglantes qu'elles en deviendront vite de véritables catachrèses ("dent ", "pointe", "aiguille") ", ils sont les inventeurs de ces analogies qui constitueront un réservoir de lieux communs pour les tableaux de montagnes du siècle suivant ${ }^{12}$. Windham compare ainsi dès 1741 les montagnes qui l'entourent à des "bâtiments d'architecture gothique "d'une hauteur vertigincuse ; Martel, quelques mois plus tard, reconnaît le premier, dans la fameuse aiguille du Dru, la forme d'un "obélisque " ou bien "une grande tour gothique, bâtie d'une pierre blanche et brune, dont les parties sont toutes fort rustiques"; Couteran, apercevant en 1775, non loin des Grands Mulets, " un roc terminé en pointe qui $[. .$.$] présentait deux faces d'un$ triangle parfait, tapissées de gazon jusqu'au sommet ", a un court instant l'illusion de "voir une des fameuses pyramides d'Égypte" 13 . Saussure, pour sa part, ne distingue dans le massif du mont Blanc que " voûtes", "arches", "gradins ", "festons ", "amphithéâtres" et "labyrinthes " 14 , Bourrit y discerne " unc forteresse avec des bastions " 15 . Quant à la séduisante image de la grotte de fées, que les voyageurs utilisent communément pour décrire la caverne glacéc de l'Arveron et qui sera popularisée plus tard par les écrivains romantiques, on la devrait à Martel ${ }^{16}$ et à Bourrit ${ }^{17}$.

Mais c'est peut-être dans la description des glaciers que ces voyageurs font preuve de la plus grande créativité, révélant par instants un vrai talent poétique et inventant des comparaisons ou des nuances que leurs successeurs romantiques ne feront que recopier platement ${ }^{18}$. Sans même revenir à l'analogie qui se trouve à l'origine de l'appellation de la Mer de glace et qui est probablement beaucoup plus ancienne qu'il n'y paraît de prime abord ${ }^{19}$, on citera simplement ici quelques-unes des descriptions qui ont motivé la pré-

11. Coxe explique ainsi l'apparition de la métaphore de l'aiguille: " D'innombrables rochers, s'effilant insensiblement depuis leur base jusqu'à leur sommet, se terminent en une pointe aigue. C'est à cette forme qu'ils doivent le nom d'Aiguilles qui les distinguent. " (ouvr. cité, t. II, p. 82).

12. Cf. la préface de Sylvain Joutr aux Vacances du lundi de Théophile Gautier (Seyssel, Champ Vallon, 1994).

13. Cf. Ph. Joutard, ouvr. cité, pp. 97, 99-100, 130. L'auteur rend toutefois justice à leurs prédécesseurs helvètes de la Renaissance, parmi lesquels Marti et Schöpf, auxquels on doit quelques images déjà fort précises : les montagnes sont par l'un qualifiées de "monuments de l'antiquité la plus ancienne ", l'autre reconnaît dans une vallée glaciaire "la forme d'une barque ou d'une arche " et dans l'Eiger "à peu près [celle d']une pyramide " (ibid, pp. 57 et 64). En 1624, Grasser, auteur d'un guide pour les voyageurs en Suisse, vantera " les masses en marbre des montagnes [qui] s'élèvent comme des murs et des tours de magnifiques édifices $"$ (ibid., p. 74).

14. Ouvr. cité, pp. 80, 88, 149-50, 177, 194, 198, 200, 203, etc.

15. Itinéraire de Genève, Lausanne et Chamouni, Genève, Didier, 1791, p. 296.

16. Celui-ci compare en effet les voûtes glacées de la caverne aux " grottes de cristal que la Fable a imaginées pour loger les Fées " (cité dans Ph. Joutaro, ouvr. cité, p. 101).

17. "[...] des crevasses embellissent cette voûte, qui rappelle à l'imagination ces peintures charmantes de grottes de fèes ; ces palais de Déesses, dont l'or, l'argent, les pierres précieuses sont la matière. " (ouvr. cité, p. 278).

18. On se référera pour mémoire aux pages merveilleuses que Ramond de CARBONNIERES leur consacre dans son commentaire du voyage de Coxe (ouvr. cité, "Observations du traducteur sur les glacières et les glaciers ", t. II, p. 96 et suiv.). 19. C'est HALLER, le célèbre auteur du poème Les Alpes (1733) qui en fait mention ("Description des Alpes " dans Les Alpes [trad. fr. par D. B. TSCHARNER], Berne, Socièté typographique, 1795, pp. 83-85). 


\section{Photo 1}

Vue du Vallais et du Rhône par M. T. Bourrit - eau forte ; $11,5 \times 19 \mathrm{~cm}, 1787$. Collection Paul Payot.

Propriété du Département de la Haute-Savoie. Consenvatoire d'Art et d'Histoire, 18 av. du Trésum 74000 Annecy.

sente recherche, comme ces lignes extraites du Voyage pittoresque aux glacières de Savoye fait en 1772 de Louis Bordier ${ }^{20}$ :

Le Glacier des Bossons offre un aspect plus imposant encore: Il dégorge plus avant dans la plaine ; les monceaux de glace sont plus considérables. L'oxil croit appercevoir tantót les vastes décombres de marbre blanc de quelque supcrbe Ville ravagée ; tantót une arméc d'énormes Tours, de sucre très-blanc, ou d'amidon transparent, rondes, quarrées, couvertes, ou sans toits, plantées les unes devant les autres à quelques pieds de distance. Le mur de glace s'élève ensuite par un talus rapide à la hauteur de huit ou neuf mille pieds. (p. 197)

Le célèbre Marc-Théodore Bourrit, chantre du mont Blanc, qui s'en accapara un temps l'exploitation touristique, n'est pas en reste ${ }^{21}$. Il présente ainsi une vue du glacier des Bois depuis le site du Chapeau dans sa Nouvelle Description des glacières et glaciers de Savoie 22 :

C'est-là où l'on voit des débris qui retracent à l'imagination une ville antique et déserte ; \& dont les palais détruits par le temps, sont admirés encore dans leurs ruines entassées, brisées dans leur chûte. Ce ne sont partout que des pilastres renversés, que des corniches,

20. Genève, Caille, 1773.

21. Cf. Sarga MousSA, "L'exotisme alpestre de Marc-Théodore Bourrit " dans L'CEil aux aguets ou l'artiste en voyage (Fr. Moureau éd.), Paris, Klincksieck, 1995.

22. Genève, Barde, 1785. 
Photo 2

La source de l'Averon par M. T. Bourrit - 87-02-142(D) - gravure aquarellée ; $25,7 \times 32,2 \mathrm{~cm}$ [vers 1780].

Collection Paul Payot. Propriété du Département de la Haute-Savoie. Conservatoire d'Art et d'Histoire, 18 av. du Trésum 74000 Annecy. cliché : D. Vidalie

des chapitcaux, des ponts à moitić rompus $\&$ mille autres configurations semblables $[\ldots]$; et tandis que l'on a les yeux fixés sur ces étonnantes productions, tout-à-coup l'on est surpris par la châte de quelques-unes de leurs parties. Ici, c'est une tour qui s'écroule ; là, une pyramide qui se brise \& tombe ; aillcurs un dome s'ćbranle f... J. (chap. X, pp. 120-21)

Dans son Itinéraire de (ienève, Lausanne et Chamouni, publić en 1791, il propose en outre ce compte rendu d'une randonnée glaciaire:

Ce glacier étoit le plus étonnant que nous eussions vu ; nous entrámes dans des défilés étroits et si profonds que les parois de chaque côté s'élevoient de quatre à cing cents pieds de haut, et après ces détroits nous nous trouvions subitement dans de grandes places, decorées d'obélisques, de la plus grande beauté, dont malgré notre fatigue nous aimions faire le tour : ces grands objets sont au-dessus de toute exagération. Nous parvinmes encore dans un labyrinthe non moins extraordinaire; d'horribles enfoncèmens étoient les scules issues qui s'offroient à nous, et notre effroi égaloit ces horreurs, dans ces momens surtout où d'énormes tours sembloient menacer ruine ; enfin, ici j’atteste le témoignage des guides, nous passámes sous des ares de triomphe bien supéricurs en grandeur à ceux bátis par la main des hommes, et ces arcs étoient d'un azur le plus transparens. Comment pouvoir sarracher à de semblables beautés ? (ouvr. cité, pp. 249-50) 
Vernes de I.uze, une quinzaine d'années plus tard, trouvera des accents voisins pour évoquer le glacier des Bossons dans son Voyage aux glaciers des Alpes de 1807 :

Dans une enceinte d'environ deux lieues de circonférence, d'immenses glaces tournées en colonnes, en obélisques, en pyramides de toutes les formes, s'élèvent par gradins sur un plan incliné rangées dans un ordre presque symétrique et vont enfin se perdre dans les nues, en réfléchissant dans leurs têtes transparentes l'azur du ciel, ou l'or vairé des nuages. Telle apparaitrait une ville de cristal dont les dimensions colossales égaleraient dans toute son architecture, celle des pyramides d'Égypte; qu'on se la figure s'élevant sur un plan que l'ceil embrasserait d'un regard, et resplendissante dans tout son ensemble des rayons du soleil qui s'y réfléchissent, s'y brisent, s'y nuancent de mille coulcurs, sur mille et mille foyers, et l'on se formera une faible idée de ce que nul mot ne peut exprimer, comme nul pinceau ne peut peindre ${ }^{23}$.

Hugo reprendra ces images, en les transfigurant comme à son habitude, dans le court Fragment d'un voyage aux Alpes ${ }^{24}$, où il narre l'excursion qu'il entreprend en 1825 en compagnie de son épouse, de sa fille Léopoldine et de la famille Nodier et où il compare les "énormes prismes de glace" de ce même glacier des Bossons d'abord aux "trois mille pierres" de Carnac, "bizarrement rangées dans la plaine ", puis à

une ville d'obélisques, de cippes, de colonnes et de pyramides, une cité de temples et de sépulcres, un palais bâti par des fées pour des âmes et des esprits ; et je ne m'étonne pas que les primitifs habitants de ces contrées aient souvent cru voir des êtres surnaturels voltiger entre les flèches de ce glacier à l'heure où le jour vient rendre l'éclat à l'albâtre de leurs frontons et ses couleurs à la nacre de leurs pilastres. (p. 563)

Ces cinq extraits ont été retenus parce qu'ils semblent pousser dans ses derniers retranchements cette logique de l'analogie architecturale qui, on l'a vu, agrémente la plupart des récits de voyage dans les Alpes à la fin du XVIII' et au début du XIX“ siècles. Si en effet les séracs représentent autant de tours, de pyramides, de pilastres, de frontons et d'arcs de triomphe, comment ne pas être tenté de filer la métaphore et d'y voir l'image d'une ville ou d'une cité, fût-clle détruite ou ruinée ? Bien sûr, il faut faire la part de la vocation indubitablement pédagogique de ces analogies, qui familiarisent le lecteur citadin avec un réel inconnu de lui et par trop déconcertant, atténuant ainsi le choc de la nouveauté : c'est là le propre de tout récit de voyage. Bien sûr, il faut également tenir compte des inévitables emprunts et jeux intertextuels qui caractérisent ce genre si particulier, même s'il reste difficile d'en mesurer la part véritable: on se contentera d'affirmer que la comparaison, la métaphore y ont aussi une fonction ornementale. Mais on peut tout de même s'étonner de retrouver dans ces représentations imagées, sinon imaginaires, de la haute montagne sous la figure d'une ville, des vestiges d'un monde aussi radicalement étranger à "ces immenses déserts des Alpes " qu'en quelques formules magistrales, Ramond de Carbonnières oppose aux "solitudes de nos plaines " 25 .

Ce choix esthérique est d'autant plus surprenant que les voyageurs cités n'accordent qu'une attention limitée, voire dédaigneuse, aux humbles agglomérations urbaines placées sur la route ou aux avant-postes des merveilles qu'ils s'apprêtent à découvrir. Si

23. Cf. CI. Lacoste-Vesseyre, ouvr. cité, t. I. pp. 53-54.

24. Dans Euvres complètes, Paris, Club français du livre, 1970, t. 2.

25. Ouvr. cité, pp. 127-28. 
Bourrit fait preuve d'une discrétion proche de l'euphémisme lorsqu'il évoque Bonneville ou Cluses ${ }^{26}$, il n'en est pas de même pour Bordier, qui, après avoir fait l'apologie de Genève, proclame nettement son mépris pour Thonon ("ville assez grande et assez sale"), Evian ("un gros vilain bourg, assez pauvre, situé dans le plus beau Pays du Monde.") ou Martigny ("I a Ville est hideuse, les Maisons mal baties, les rues infectes. "), sans parler des propos peu amènes qu'il a pour l’aspect général de Bonneville, les habitants de Sallanches ou les "Grands Domes de bois enfumés" de Cluses ${ }^{27} \ldots$

Pourquoi donc ces voyageurs cherchent-ils à retrouver dans cette nature inviolée, inhospitalière, vierge de toute présence humaine et justement convoitée comme telle, ce qui est précisément la forme la plus accomplic de la civilisation : l'agglomération urbaine? Pourquoi ces citadins des lumières et du romantisme projettent-ils sur les paysages sublimes de la haute montagne ces marques imaginaires d'un paysage urbain alors en pleine expansion, au moment méme où les villes acquièrent plein droit de cité en littérature?

On aura bien sûr noté tout ce que ces éléments monumentaux, pilastres, chapiteaux, corniches, colonnes, pyramides, obélisques ou arcs de triomphe, ont de commun avec l'architecture colossale et utopique d'un Ledoux ou d'un Boullée: comment ne pas retrouver, dans le parallèle tracé par Vernes de Luze entre les glaciers et les pyramides de l'Égypte, l'esprit qui anime les projets, hélas restés à l'état de plans, du cénotaphe de Newron ou du "monument destiné aux hommages à l'Etre suprême "28?

Mais comment ne pas être sensible non plus à cette commune fascination pour la ruine, surtout lorsque celle-ci atteint des proportions qui dépassent la raison humaine? L.es analogies urbaines que font surgir les glaciers des Alpes dans l'imagination de ces voyageurs renvoient en effet à des villes qui disposent du double et fascinant pouvoir d'être à la fois irréelles, par leur caractère strictement figuré, leur situation, leurs proportions et leurs matériaux, et en ruine, donc porteuses de cette potentialité de réverie mélancolique si chère à la sensibilité retrouvée d'un XVIII' siècle à l'agonie. Autant dire que le glacier donne au spectateur imaginatif la représentation de la "cité idéale ", non plus au sens où l'entendait la pensée renaissante, dynamique et visionnaire, d'un Piero della Francesca, mais sous cette forme "ruineuse", au sens vieilli du terme, alliant avec bonheur pouvoir de la nature et vestiges de la culture, qui entraîne l'âme, bien mal nommée "néoclassique" ou "préromantique", vers les voluptueux abimes de la méditation.

Pourtant, ces ruines imaginaires, contrairement à leurs homologues de l'Antiquité, ne renvoient le spectateur à aucun passé: elles sont en quelque sorte des décombres à l'état

\footnotetext{
26. "Le premier coup-d’œil sur la Bonneville en donne une idée avantageuse : on y voit une grande place, où l'on devroit planter des arbres, qui, en l'embellissant, la garantiroient des chaleurs excessives qu'on y éprouve, \& contribueroient à la salubrité de l'air. [...] De Songi, qui est à demi-lieue de Cluse, l'on ne découvre pas encore cette ville : la beauté du paysage contribue aussi à la faire oublier $[\ldots]$ elle seroit oubliée des gens aisés, qui passent la plus grande partie de l'année dans leurs campagnes, s'ils n'y étoient rappelés par des fêtes patriotiques. " (Nouvelle Description [...], ouvr. cité, pp. 7 et 9-11).

27. Ouvr. cité, pp. 15-20, 26, 37, 152, 288, 293, 296

28. Cf. entre autres Jean-Marie Perouse de Montclos, Etienne-Louis Boullée, Paris, Flammarion, 1994.
} 
Photo 3

Vue du Mont-Blanc, du Bionnassay, du Trelatete, du Pic du Col de la Seigne depuis le passage du Bonhomme par Jean-

Antoine Linck - INC059 - crayon noir ; $44,3 \times 57,5 \mathrm{~cm}$. Collection Paul Payot. Propriété du Département de la Haute-Savoie. Conservatoire d'Art et d'Histoire, 18 av. du Trésum 74000 Annecy. cliché : D. Vidalie

absolu, des quintessences de ruines, où toute signification historique est absente - comme elle semblait, aux yeux des contemporains, s'être à jamais retirée des pyramides égyptiennes. Bien plus, ces "images de ruines" se manifestent au voyageur dans le processus même de leur évolution: Bourrit ne manque pas de montrer à son lecteur ces tours qui "menace[nt] ruine" ou "qui s'écroule[nt]", cette "pyramide qui se brise et tombe", ce "dôme " qui "s'ébranle ". A la manière d'un prodigicux kaléidoscope, la nature détruit sous les yeux mêmes du spectateur le tableau que celui-ci venait à peine d'admirer, lui dévoilant en quelque sorte les coulisses de la Création et les processus dans lesquels, infime et éphémère poussière, il se trouve lui-même impliqué.

Le message des fausses ruines que le voyageur croit déceler dans les glaciers est donc d'un tout autre ordre qu'historique. Il dépasse même les considérations que peut faire l'homme sur son destin au milieu des ruines antiques, comme le souligne Wilhelm Heinse en 1780 :

C'est d'un âge antique, des ruines de la Création, que je vous écris, cher Père Gleim ; aux côtés de ces ruines, celles de la Grèce et de Rome paraissent à peine comme de petits châteaux de cartes effondrés chers aux jeunes enfants. [...] Très cher ami, la fin du monde se trouve bel et bien ici. Le Gothard est un véritable cimetière de la nature. A la place des ossements des morts, repose ici une prodigieuse quantité de massifs désertiques et, dans les vallées profondes, s'entassent les décombres des roches. [...] J'ai vu le début et la fin du 


\section{Illustration non autorisée à la diffusion}

\section{Photo 4}

Vue de la source de l'Arveyron, dessiné par Bélanger 87-02-192 (D) - eau forte ; $48 \times 68 \mathrm{~cm}$

Collection Paul Payot. Propriété du Département de la Haute-Savoie. Conservatoire d'Art et d'Histoire, 18 av. du Trésum 74000 Annecy. cliché : D. Vidalie

monde, et, pour la première fois, j’ai contemplé les choses dans leur véritable lumière ; j’ai pénétré avec ravissement jusque dans l'harmonic la plus intime et la plus secrète des êtres, et mon cour, mon esprit et tous mes sens ont été baignés d'extase. 20)

Dès 1709 , Shaftesbury analysait ainsi les raisons qui poussent ses congénères à braver tous les dangers pour aller contempler les spectacles offerts par la haute montagne:

I à, transportés par des objets si nouveaux, les hommes insensés acquièrent le don de réflexion ; ils acceptent de considérer les changements continuels qui se produisent à la surface de la terre. Ils vivent, comme en un seul instant, les révolutions des áges anciens, les formes mouvantes des choses, et la décrépitude méme de notre globe. Tandis qu'ils en contemplent la jeuncesse et la formation primitive, ils aperçoivent, dans les destructions visibles et les irréparables brèches de la montagne ravagée, que le monde lui-même est une noble ruine dont le terme est proche. ${ }^{30}$

Est-il alors si étonnant que ces images de cités antiques et ravagées coexistent, chez Hugo ou chez Heinse, avec celles de la nécropole et du tombeau? Il est donc des lieux où l'homme n'a pas sa place et qui ne peuvent en outre que lui rappeler l'idée de la

29. Lettres du $1^{\text {e' }}$ et du 10 septembre 1780, citées par Michaël JAKOB dans le Panorama littéraire qu'il a élaboré pour le catalogue de l'exposition "Le sentiment de la montagne " (musée de Grenoble / ERMN / Glénat, 1998). Qu'il trouve ici l'expression de ma gratitude pour les précieux conseils qu'il m'a donnés au début et dans le cours de cette recherche.

30. Cité dans Jean StaRobinski, L'Invention de la liberté (1964), Genève, Skira, 1994, p. 160. 
disparition: la sienne et celle de la nature, si fragile là-même où elle semble la plus redoutable... Mais c'est sans doute la médiation représentée par la grandeur, la pureté, les qualités esthétiques de ces mirages urbains des glaciers qui contribuent à transformer ce constat, doublement horrifiant pour la conscience humaine, en un spectacle sublime auquel, à l'instar de Bourrit, on ne saurait "s'arracher " si facilement...

Hugo et, quelques décennies plus tard, Théophile Gautier semblent tirer le bouquet final de cet étonnant feu d'artifice analogique qui, pour la plus grande joie des lecteurs, transforme la haute montagne en une féerie architecturale et urbaine auprès de laquelle le carton-pâte des Disneyland américains fait bien piètre figure. Dès le milieu du siècle, sommets et glaciers des Alpes commencent à devenir le domaine réservé des grimpeurs et des scientifiques: il faut beaucoup de bonne volonté pour trouver la moindre poésie dans les relevés de variation glaciaires réalisés par Desor, Agassiz ou le prince Roland Bonaparte pour le compte des sociétés savantes de l'époque ${ }^{31}$. Seule l'imagination fantastique d'un Lovecraft osera reprendre ces rêveries dans quelques histoires démentielles ${ }^{32}$, mais la moindre ironie de l'histoire n'est peut-être pas qu'il ait fallu attendre l'explosion de la société des loisirs et des sports d'hiver, en ce XX' siècle finissant, pour que sortent de terre, sous une forme certes trop souvent prosaïque et dérisoire, ces "villes de cristal " qu'un obscur voyageur de l'Empire crut apercevoir un jour dans un glacier...

31. On rappellera simplement pour mémoire les Excursions et séjours dans les glaciers et les hautes régions des Alpes, de M. Agassiz et de ses compagnons de voyage, par E. DESOR (Neuchâtel-Paris, Kissling-Maison, 1844 et 1848) et, entre autres, Les Variations périodiques des glaciers français (...) du prince Roland Bonaparte (Paris, Chamerot et Renouard, 1891).

32. Cf. en particulier At the Mountains of Madness, 1931. 